2. Hyperbola $x y=$ const. (Fig. 2)

$$
\begin{gathered}
\frac{1}{x} \frac{d x}{d s}+\frac{1}{y} \frac{d y}{d s}=0 . \\
\therefore \quad P T=-P t .
\end{gathered}
$$

3. Conic $\beta \gamma=k \alpha^{2}$, having $A B, A C$ tangents and $B C$ chord of contact. (Fig. 3)

hence

$$
\begin{gathered}
\frac{1}{P M}+\frac{1}{P N}=\frac{2}{P L} \\
(M N, P L)=-1 .
\end{gathered}
$$

4. Cubic Hyperbola $\alpha \beta \gamma=\alpha^{\prime} \beta^{\prime} \gamma^{\prime}$ through $P\left(\alpha_{1}, \beta_{1}, \gamma_{1}\right)$. (Fig. 4)

- $2 P L=$ harmonic mean between $P M, P N$.

Draw $A Q$ the fourth harmonic mean to $A B, A P, A C$ and a parallel to $B C$ at three times the distance $P$ has to $B C$. The intersection $U$ of these lines gives the tangent at $P$, for

$$
P U=\text { harmonic mean of } P M, P N=-2 P L
$$

5. Similar results apply to curves $a \gamma=k \beta \delta: O P^{2}=k P U . P U^{\prime}$ (where $O$ is a fixed point and $P U, P U^{\prime}$ are perpendiculars on fixed straight lines) ; $27 a y^{2}=4 x^{3} ;(x+y+z)^{3}=6 m x y z$, etc.

R. F. Davis.

\title{
Geometrical Proof of a Trigonometrical Identity.-
} The following method of proof of the identity,

$$
1-\cos ^{2} A-\cos ^{2} B-\cos ^{2} C-2 \cos A \cos B \cos C=0
$$

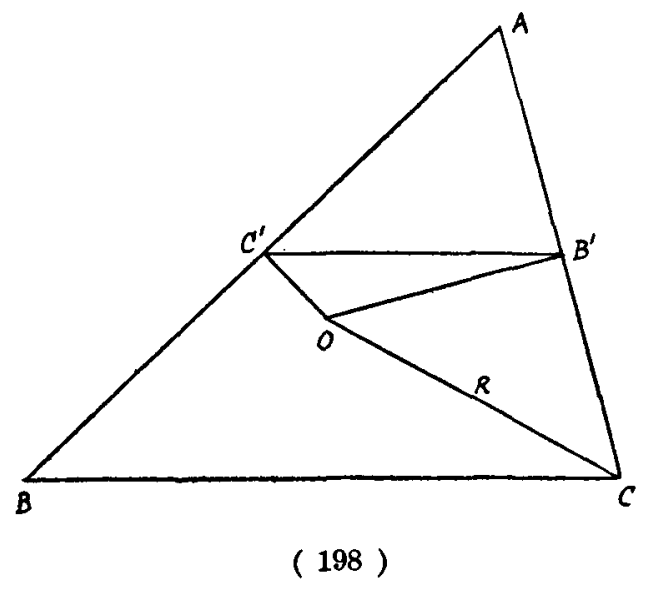


where $A, B, C$ are the angles of a triangle, was proposed in an examination paper, and it has been suggested to me that it might be of interest to teachers. It is rather easier than that given on p. 344 of "New Trigonometry for Schools" by Lock and Child.

$O$ is the circumcentre of the triangle $A B C$ and $B^{\prime}$ and $C^{\prime}$ are the mid points of $A C$ and $A B$. From the triangle $O B^{\prime} C$ since $\angle C O A=2 B, O B^{\prime}=R \cos B$. Similarly $O C^{\prime}=R \cos C$.

$$
B^{\prime} C^{\prime}=\frac{a}{2}=R \sin A \text {. }
$$

But from the triangle $B^{\prime} C^{\prime} O$, since $\angle B^{\prime} O C^{\prime}=180^{\circ}-A$,

$$
\begin{aligned}
B^{\prime} C^{\prime 2} & =O B^{\prime 2}+O C^{\prime 2}+2 O B^{\prime} . O C^{\prime} \cos A . \\
\therefore \quad \sin ^{2} A & =\cos ^{2} B+\cos ^{2} C+2 \cos B \cos C \cos A
\end{aligned}
$$

which gives the result.

R. J. T. BeLL.

Note on students and original work. - I have sometimes been laughed at when I have ventured the opinion that University students of mathematics ought to do original work in mathematies as part (and an important part) of their degree work. But this will not prevent me from maintaining that original work should be done by every scholar in a junior class of geometry. Of course in one sense this is a mere platitude, for every "problem" and "rider" requires for its solution original work on the part of the scholar. $\mathrm{He}$ or she that would solve a geometrical problem must "intend the mind continually" on it, like Sir Isaac Newton, and acquire the faculty of trying different points of view till the right one is found. For him or her, at any rate, the Pragmatists' test of truth is appropriate. He asks of every hypothesis "will it work?" and finds the answer by actual trial.

But besides problems and riders, why not "Dissertations"? The following little exercise has been used with good effect in a junior class of geometry.

Consider the following properties of the parallelogram $A B C D$ :

(1) $A B$ parallel to $C D$

(2) $A D$ parallel to $B C$

(3) $A B=C D$

(4) $A D=B C$ 